\title{
Microplates for Crystal Growth and in situ Data Collection at a Synchrotron Beamline
}

\author{
Miao Liang ${ }^{1,2} \mathbb{D}^{-}$, Zhijun Wang ${ }^{1,3}$, Hai Wu ${ }^{1,2}$, Li Yu ${ }^{1,2}$, Bo Sun ${ }^{1,3}$, Huan Zhou ${ }^{1,3}$, Feng Yu ${ }^{1,3}$, \\ Qisheng Wang ${ }^{1,3, * \mathbb{D}}$ and Jianhua $\mathrm{He}^{1,4, *}$ \\ 1 Shanghai Institute of Applied Physics, Chinese Academy of Sciences, Shanghai 201800, China; \\ liangmiao@sinap.ac.cn (M.L.); wangzj@sari.ac.cn (Z.W.); wuhai@sinap.ac.cn (H.W.); yuli@sinap.ac.cn (L.Y.); \\ sunbo@zjlab.org.cn (B.S.); zhouhuan@zjlab.org.cn (H.Z.); yufeng@zjlab.org.cn (F.Y.) \\ 2 University of Chinese Academy of Sciences, Beijing 100049, China \\ 3 Shanghai Synchrotron Radiation Facility, Shanghai Advanced Research Institute, Chinese Academy of Sciences, \\ Shanghai 201204, China \\ 4 The Institute for Advanced Studies, Wuhan University, Wuhan 430072, China \\ * Correspondence: wangqisheng@sinap.ac.cn (Q.W.); hejianhua@whu.edu.cn (J.H.)
}

Received: 30 July 2020; Accepted: 6 September 2020; Published: 9 September 2020

\begin{abstract}
An efficient data collection method is important for microcrystals, because microcrystals are sensitive to radiation damage. Moreover, microcrystals are difficult to harvest and locate owing to refraction effects from the surface of the liquid drop or optically invisible, owing to their small size. Collecting X-ray diffraction data directly from the crystallization devices to completely eliminate the crystal harvesting step is of particular interest. To address these needs, novel microplates combining crystal growth and data collection have been designed for efficient in situ data collection and fully tested at Shanghai Synchrotron Radiation Facility (SSRF) crystallography beamlines. The design of the novel microplates fully adapts the advantage of in situ technology. Thin Kapton membranes were selected to seal the microplate for crystal growth, the crystallization plates can support hanging drop and setting drop vapor diffusion crystallization experiments. Then, the microplate was fixed on a magnetic base and mounted on the goniometer head for in situ data collection. Automatic grid scanning was applied for crystal location with a Blu-Ice data collection system and then in situ data collection was performed. The microcrystals of lysozyme were selected as the testing samples for diffraction data collection using the novel microplates. The results show that this method can achieve comparable data quality to that of the traditional method using the nylon loop. In addition, our method can efficiently and diversely perform data acquisition experiments, and be especially suitable for solving structures of multiple crystals at room temperature or cryogenic temperature.
\end{abstract}

Keywords: microcrystals; microplate; grid scanning; in situ data collection

\section{Introduction}

In recent years, in order to efficiently obtain the structure of protein, various processing steps of the protein crystallography have been improved and optimized, especially with the development of in situ X-ray crystallography [1]. The method of in situ diffraction has been developed to directly collect datasets from the location where crystals were grown using $X$-ray diffraction, which eliminates the process of transferring the crystal sample to nylon loop and avoids the influence of human factors on the quality of the crystals. This method was originally used to screen crystals and verify the quality of crystals, but now this method is mainly used to collect multiple data sets to obtain high-resolution protein structures, because in situ diffraction can greatly improve the efficiency of data collection, and at the same time, is very suitable for some special crystals. For example, some proteins can only 
produce microcrystals, and microcrystals are fragile to transfer with a normal nylon loop. In addition to the development of in situ data collection methods, microcrystals are sensitive to radiation damage, so it is necessary to collect data from multiple crystals in a small wedge and integrate the diffraction images of multiple crystals into a full dataset. Since the determination of radiation-sensitive crystal structures requires a large number of crystals, high-throughput data collection methods are also needed to improve efficiency. A number of successful devices have already been designed for in situ data collection at different light sources. So far, microfluidic devices [2-4], chips [5], and regular 96-well crystallization plates [6] are the main methods reported for in situ data collection.

Microfluidic devices including capillaries and nano-droplets are usually used for protein crystallization and then directly for in situ data collection at room temperature using such devices. Microfluidic devices provide sufficient convectionless space for high-throughput crystal growth and screening. For example, Pinker et al. reported a microfluidic device ChipX [7] that can, not only obtain high-quality crystal and diffraction data, but also perform in situ characterization without directly processing crystals. Furthermore, the microfluidic device ChipX is good for in situ data collection for fragile crystals. However, ChipX is not suitable for flash-cryocooling of crystals, which will cause the liquid to freeze. In addition, the microfluidic device based on capillary is also used for in situ data collection of protein crystals [8]. It has been reported that two sets of devices containing aqueous solution and oil respectively are designed to cooperate with the capillary [9]. The aqueous solution including the protein solution and precipitant is used for protein crystallization, and the oil is used to separate the aqueous solution to form a separate crystallization environment, and then the microcapillary containing the crystal is directly installed on the beamline station for data collection after the crystallization experiment. The in situ method based on nano-droplets is also generally implemented by the microfluidic device [10]. The crystals in the capillary gradually move to the surface of the nano-droplets and are fixed near the droplet interface. During the whole process, there is no special operation to fix the crystals, crystals are fixed by the high surface tension of the droplet and used for further data collection. Furthermore, the in situ method based on nano-droplets can realize free interface diffusion crystallization and large-scale preparation of monodisperse crystals, which avoids crystal accumulation. However, the data is collected in the liquid phase at room temperature so that the crystal is susceptible to radiation damage [11].

Chip and film were reported to transfer or grow crystals, and then mount them on the goniometer head for data collection. These methods do not limit the size of crystals. It has a high hitting rate of $\mathrm{X}$-ray during data collection. The amount of protein used is small and only a few hundred crystals are needed to obtain a full dataset of protein structures [12-15]. The chip material generally uses quartz with high light transmittance and low background scattering [16]. X-rays have a high hitting rate to the crystal using a chip-based sample delivery method. Using micro-nano processing technology, holes or grooves are etched on the chip, and then the crystal is fixed in the holes and grooves on the chip for diffraction data collection. Zarrine-Afsar et al. first proposed the application of the chip-based sample-delivery method for protein crystal data collection [17]. They grew the crystals in situ on a chip with a polyimide film attached to the lower end. The chip has a grooved array (silicon mesh). By adding glass beads in the grooved area of the chip, the random orientation of the crystals is induced by increasing the roughness. The chip can be used for the in situ growth of macromolecular crystals and serial data collection. When data is collected, it is allowed to rotate at a certain angle, and collect multiple sets of diffraction data for one crystal. The sample consumption is small and it is increased by adding glass beads. The surface roughness makes the crystals oriented randomly. Some structures have been obtained by this method. However, there are chip grids, polyimide films, and crystalline solutions that will cause high background scattering. A thin film, transferred with crystals, fixed to the goniometer head for diffraction data collection was reported by Li et al. [18]. Li et al. designed a bracket, and then attached the polyimide membrane to the resin bracket with SuperGlue. The microcrystals were transferred from the coverslip to the surface of the polyimide membrane by a micromesh loop, a glass capillary with a tip diameter less than $100 \mu \mathrm{m}$ was used to remove excess liquid, and then 
crystals were sealed with another polyimide film to protect it from dehydration, but human factors are added during the sample transfer. Baxter et al. designed a high-density grid [19], covered with a polymer film or sleeve, for efficient data collection for multiple crystal samples, incubation chambers have been developed to support crystallization experiments on grids, but the grid cannot screen the crystallization conditions because there is only one type of desiccant in the incubation chamber.

Currently, a particular research interest is the possibility of data collection directly from crystallization plates, normal size 96-well plates are reported for crystal screening and in situ data collection [20]. With the development of various systems for plate setup and handling, the crystallization plates have been standardized to achieve compatibility with some beamline platforms, for example, the Structural Biology Center (SBC) beamline 19-ID, located at the Advanced Photon Source, USA. Significant progress has been made to provide plates with a low $\mathrm{X}$-ray absorption profile and scattering properties, such as the MiTeGen In situ-1 Plate (MiTeGen, Inc.). However, with these kinds of methods, it is difficult to focus and align the crystals to the beam position once the plates rotate to a small wedge angle.

Here, a set of simple and inexpensive microplates (plate A and B) is designed for screening crystallization conditions and in situ data collection at room temperature or cryogenic temperature. Plate A is used for setting drop vapor diffusion crystallization. The assembly of plate B and a specially designed crystallization plate is applied for the hanging-drop vapor-diffusion method. The $12.5 \mu \mathrm{m}$ thick Kapton membranes are used for crystal growth and sealing up the microplates. Lysozyme is used as the model protein for crystal growth, and lysozyme crystals are used to verify the practicability of the new device and method. Structure obtained by in situ method is compared with that obtained by the traditional method. The grid scanning, implemented from Blu-Ice [21] was used at the Shanghai Synchrotron Radiation Facility (SSRF) BL18U1 beamline for sample location and data collection. Results show that the devices can be used for screening crystallization conditions and in situ data collection from multiple crystals.

\section{Materials and Methods}

\subsection{Design for Microplates}

As shown in Figure 1, plate A and B are manufactured using a three-dimensional printer. The size of the microplate depends on the limitation of the goniometer and the spray area of the liquid nitrogen nozzle. The diameter of the spraying area of the liquid nitrogen nozzle is $6 \mathrm{~mm}$ at BL18U1, and the $\mathrm{Y}$-axis limitation of the goniometer is $\pm 3 \mathrm{~cm}$. In order to enable the crystal samples in the microplate to be moved to the X-ray optical path, and the crystal samples to be covered by liquid nitrogen cooling gas, the size of the microplate is designed to be less than or equal to $20 \mathrm{~mm}$. Plate A has five crystallization chambers, and each crystallization chamber is composed of two protein wells and a reservoir well, and the common space is designed in the crystallization chamber to support sitting vapor-diffusion crystallization experiments. Each protein well and reservoir well are hollowed out (Figure 1a), and the position of the bottom column is on the side of the protein wells to ensure that the bottom center of plate A and the bottom center of the protein wells are on a straight line (defined as the centerline). It is worth noting that the distance between any point of the bottom of protein wells and the centerline does not exceed $1 \mathrm{~mm}$. The reason for this is that the deflection of plate A does not cause the crystal to deviate from the optical path during data collection. The size of the bottom column is designed to match the magnetic base to be stably fixed on it, and the size of the reservoir wells can be changed according to different crystallization conditions. Plate A is sealed by two Kapton membranes for sitting-drop vapor-diffusion crystallization experiments after loading the protein samples. 

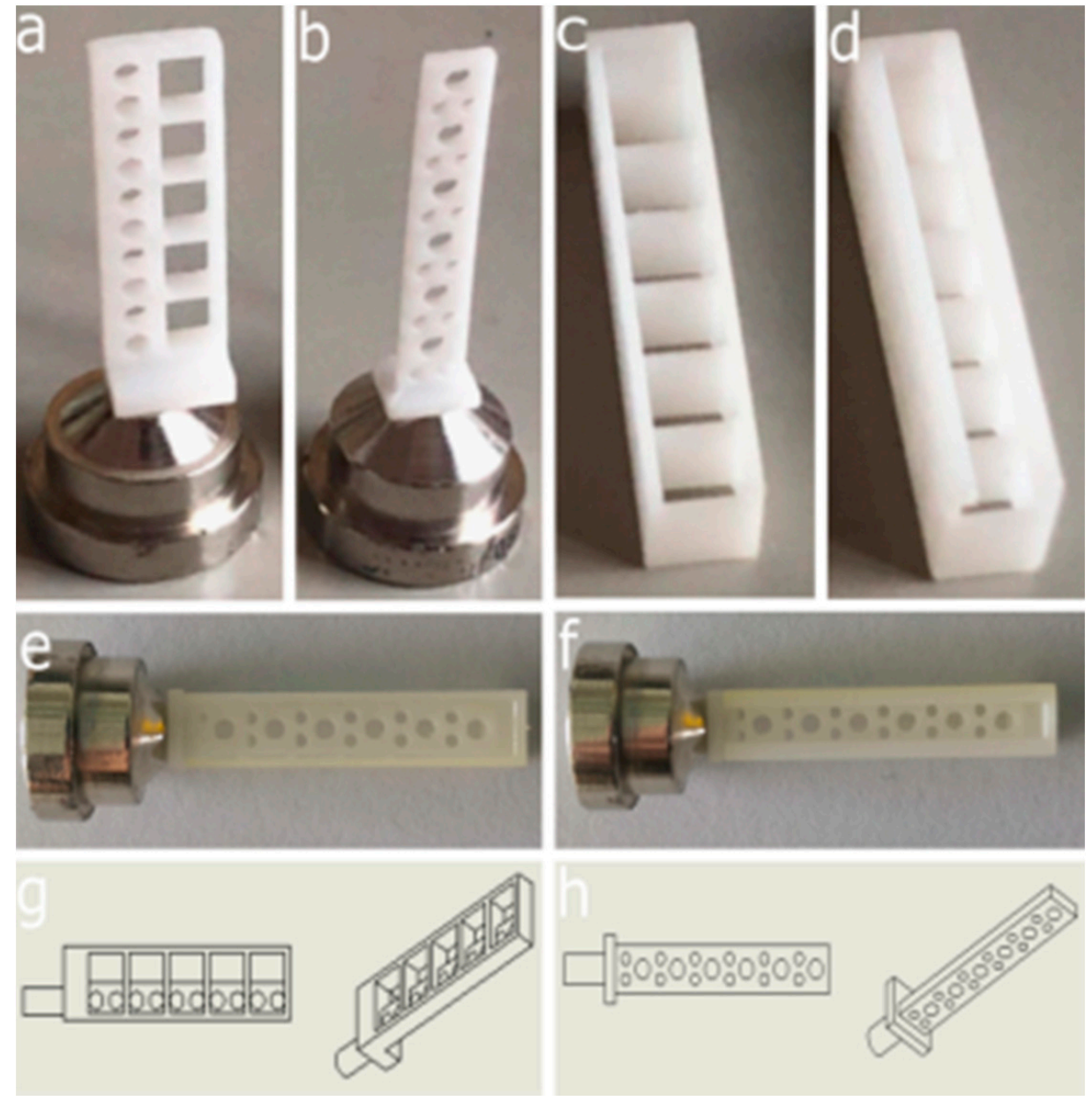

Figure 1. (a) Crystallization plate (Plate A) used for sitting-drop vapor-diffusion crystallization experiments; (b) Crystallization plate (Plate B) used for hanging-drop vapor-diffusion crystallization experiments; (c) Incubation chamber for Plate B by covering, crystallization buffers loaded into each chamber for screening of crystallization conditions for Plate B; (d) Incubation chamber for Plate B by insertion. (e) Assembly image for Plate B with incubation chamber in Figure 1c; (f) Assembly image for Plate B with incubation chamber in Figure 1d; (g) three-dimensional device structure of plate A; (h) three-dimensional device structure of plate B.

Plate B has six large protein wells and twelve small protein wells (Figure 1b). Incubation chambers (Figure 1c,d) are hollowed out. The hollowing of the reservoir well not only provides a preliminary observation of the crystal through the microscope but also facilitates the cleaning and secondary use of the plate. The size of the protein wells (Figure $1 \mathrm{~b}$ ) can be selected to fit different experimental settings, the bottom center of plate B and the bottom center of large protein wells are on a straight line (defined as the centerline), and the distance between any point of the bottom of the protein wells and the centerline does not exceed $1 \mathrm{~mm}$. Unlike plate A, plate B utilizes a specially designed crystallization plate in the crystallization experiment. The Kapton membrane only needs to seal the side where the centerline is. After loading the protein samples onto plate B, then plate B was assembled with the incubation chambers (Figure 1c,d) for hanging-drop vapor-diffusion crystallization experiments (Figure 1e,f). There are six reservoir wells in the incubation chambers (Figure 1c,d), which is sealed by the Kapton membrane at the bottom side before loading the crystallization buffers into each well. 


\subsection{Crystal Growth}

Chicken egg white lysozyme microcrystals smaller than $20 \mu \mathrm{m}$ were used as testing samples. We used the controlled variable method to explore the crystallization conditions to grow microcrystals. For plate A (sitting-drop vapor-diffusion crystallization), the side of the centerline of plate A needs to be sealed with a Kapton membrane before the crystallization experiment, and then $0.8 \mu \mathrm{L}$ of crystallization drops were added to each protein well and $8 \mu \mathrm{L}$ of reservoir solution was added to each reservoir well. The lysozyme crystallization drops are prepared by dissolving $10 \mathrm{mg} / \mathrm{mL}$ of lysozyme protein in a reservoir solution. The reservoir solution is a solution with a concentration of $0.2 \mathrm{M}$ citric acid and $0.2 \mathrm{M}$ sodium acetate at a $1: 1$ ratio, $12 \%(\mathrm{w} / \mathrm{v}) \mathrm{NaCl}$, and then a mix $0.4 \mu \mathrm{L}$ of a protein sample and $0.4 \mu \mathrm{L}$ of a reservoir solution. Finally, the other side of plate A was sealed, lysozyme microcrystals were grown with the sitting-drop vapor-diffusion method, and microcrystals appeared after $6 \mathrm{~h}$ of incubation at $291 \mathrm{~K}$. For plate B (hanging-drop vapor-diffusion method), the crystallization drops and reservoir solution used were the same as plate $\mathrm{A}$. The side of the centerline of plate $\mathrm{B}$ also needs to be sealed with a Kapton membrane before the crystallization experiment, and then $0.8 \mu \mathrm{L}$ of crystallization drops comprised of $0.4 \mu \mathrm{L}$ of a protein sample and $0.4 \mu \mathrm{L}$ of a reservoir solution were added to each large protein well and $0.4 \mu \mathrm{L}$ of crystallization drops comprised $0.2 \mu \mathrm{L}$ of a protein sample and $0.2 \mu \mathrm{L}$ of a reservoir solution were added to each small protein well. The bottom of the crystallization plate was sealed with a Kapton membrane and $40 \mu \mathrm{L}$ of reservoir solution was added to each reservoir well. Finally, plate B was assembled with the incubation chambers (Figure 1c,d) by covering or inserting, and lysozyme microcrystals were grown with the hanging-drop vapor-diffusion method. Microcrystals appeared after $6 \mathrm{~h}$ of incubation at $291 \mathrm{~K}$.

\subsection{Sample Loading}

The bottom of the microplates were coated with glue and tightly fixed into the magnetic base (Figure 1a,b). For the sitting-drop vapor-diffusion experiment with plate $\mathrm{A}$, the reservoir solution in the reservoir wells frost at cryogenic temperature and affect the protein wells, therefore, in our experiment, plate A was directly mounted on the goniometer head for data collection at room temperature. During the rotation of plate $\mathrm{A}$, it is difficult for the reservoir solution and the crystallization drops to flow out due to atmospheric pressure and liquid tension in the actual test. For the hanging-drop vapor-diffusion experiment with plate $B$, we removed plate $B$ from the incubation chambers (Figure $1 c, d)$, and then directly added $0.2 \mu \mathrm{L}$ of glycerol to each large protein well and added $0.1 \mu \mathrm{L}$ of glycerol to each small protein well. Finally, the side away from the centerline was sealed with a Kapton membrane, and then plate B was mounted on the goniometer head for data collection at cryogenic temperature.

\subsection{Data Collection}

The experiment was carried out at the SSRF beamline BL18U1. The energy used for data collection was $12.662 \mathrm{keV}$, the photon flux at the sample point was $6 \times 10^{11} \mathrm{phs} / \mathrm{s}$, and the size of focused beam used for experiment was $20 \times 20 \mu \mathrm{m}^{2}$.

Crystals in the microplates were aligned to beam position before collecting data at room temperature or cryogenic temperature. Firstly, the edge of the membrane was aligned to beam position at low magnification and then the microplate was rotated by $90^{\circ}$ to locate the side of the centerline. Further steps were performed to bring the protein well to the beam and make the crystals align to beam position at high magnification. At room temperature, we used the optical method to locate lysozyme microcrystals due to high radiation damage, grid scanning was used to locate lysozyme microcrystals due to the protection of cryoprotectant at cryogenic temperature. The scanning area and the type of grid scanning were selected, BluIce system automatically scanned each grid point in the area with low-dose X-rays after setting the parameters, and then automatically calculated the initial diffraction value of each grid point and draw the diffraction pattern. Datasets were collected with a small wedge angle for each crystal. 
Radiation damage is a factor that must be considered for data collection at room temperature or low temperature. X-rays will affect the life of the crystal. The life of the crystal is considered as the radiation dose that the crystal can receive without structural changes. Radiation damage is divided into overall radiation damage and local radiation damage. The overall radiation damage is not for a specific atom, it is mainly manifested in the diffraction pattern, which makes the resolution of protein crystals decrease, especially the high-resolution shell. Local radiation damage is the direct inelastic scattering of X-rays with the sample, through light absorption or Polly Compton scattering. Local radiation damage breaks some covalent bonds of protein molecules, such as disulfide bonds, which appear as a small group of atoms in the electron density map. Local radiation damage can cause researchers to misunderstand the crystal structure. The lifetime of the crystal depends on many factors, such as luminous flux density, wavelength, and protein sample composition (including molecular weight, heavy atom content). The absorption of X-ray by heavy atoms is higher than that of other atoms. Regarding the overall radiation damage, Owen et al. [22] proposed that the decrease in the diffraction quality of protein crystals produced by synchrotron radiation and the local changes in the protein structure can be measured by radiation dose. Local radiation damage depends on many factors, such as the folding of protein structures. This kind of radiation damage is difficult to judge from the diffraction quality, and can only be found by analyzing the structure. According to the radiation dose limit, we collected five diffraction images at room temperature and 40 diffraction images at cryogenic temperature. The exposure time for both was set to $0.5 \mathrm{~s}$ with a degree increment of $1^{\circ}$.

\subsection{Data Processing and Analysis}

Data collection at room temperature or cryogenic temperature required the use of multiple crystals to obtain a complete set of data. The crystals were screened on plates A and B, 20 crystals were selected at room temperature and 10 crystals were selected at cryogenic temperature for data collection. Each dataset was indexed by XDS [23], and then imported into the BLEND program [24] in CCP4 [25], and then we combined each dataset for integration and homogenization. We selected the best combination of data from several sets of data according to the quality of the data. The method of structure determination was adopted from the molecular replacement program CCP4, the search model of lysozyme was from the PDB1rcm of the protein database, and then Phenix [26] was used to refine the structure. The final model was modified by Coot [27].

\section{Results and Discussion}

\subsection{Crystal Growth in Microplates}

A typical result of crystal growth in microplate A with sitting-drop vapor-diffusion crystallization is shown in Figure 2a. Crystals can be clearly viewed from our beamline on-axis-video (OAV) system in Figure $2 \mathrm{~b}$. Conversely, crystal growth in microplate $\mathrm{B}$ with the hanging-drop vapor-diffusion method can also be achieved (Figure 2c,d). Using different desiccant in the reservoir well, we can grow different crystals in the microplates, which allows us to directly screen the crystallization conditions by the beamline video system. A typical result of the crystal growth under different crystallization conditions is shown in Figure 2e,f, which shows that crystallization conditions can be initially screened with microplate A and B under the microscope system (Figure 2e,f). Moreover, the suspension points or wells would be screened with grid scanning for further confirmation. 

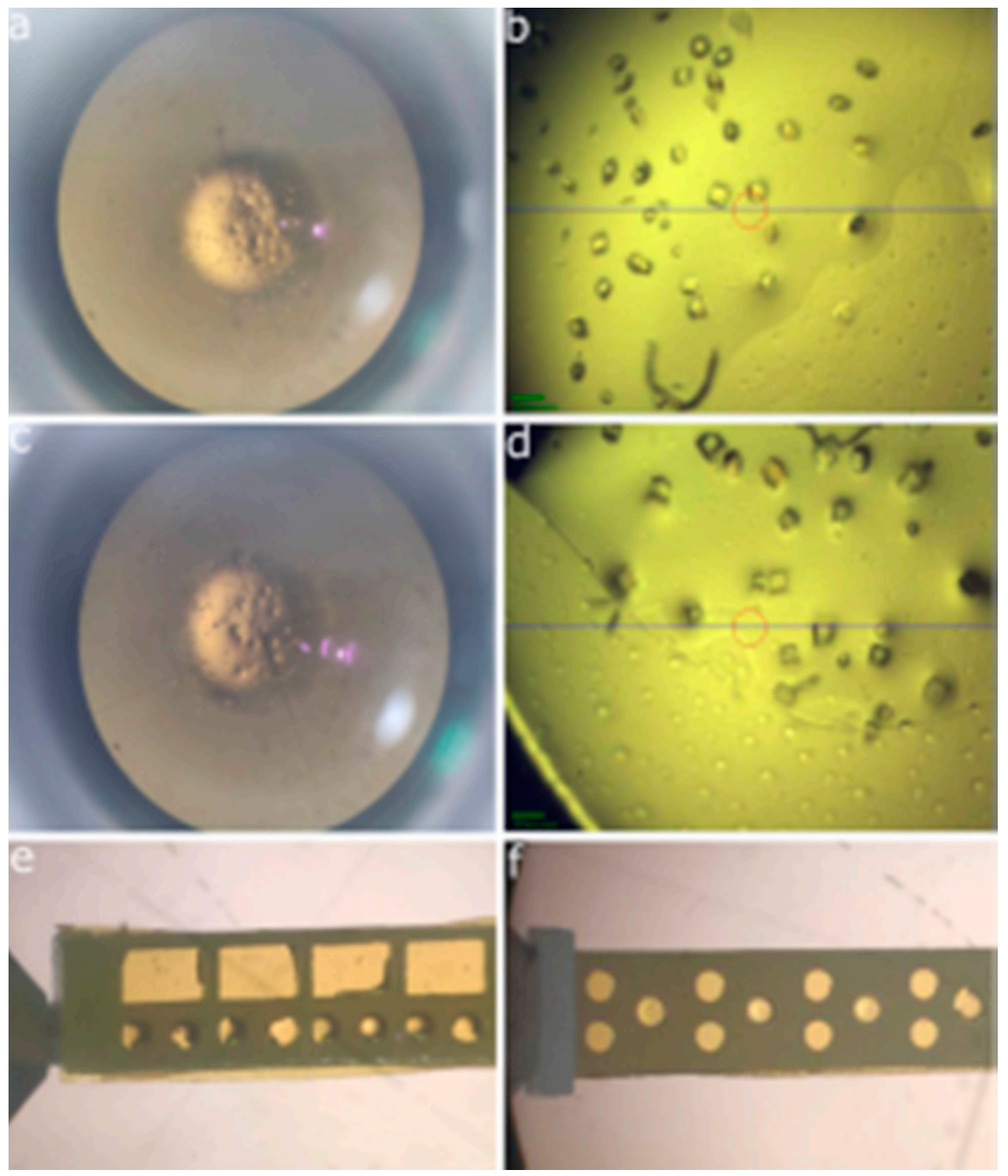

Figure 2. (a) Crystallization experiments, viewed under low magnification of the microscope, with plate A, the Kapton membrane is fixed on both sides of plate A to support crystallization experiments. (b) The growth of lysozyme microcrystals, viewed under high magnification of an on-axis-video system in a protein well of plate A, the length of microcrystal is less than $20 \mu \mathrm{m}$. (c) Crystallization experiments, viewed under low magnification of the microscope, with plate $B$, the Kapton membrane is fixed on the side where the centerline is of plate B to support crystallization experiments. (d) The growth of lysozyme microcrystals, viewed under high magnification of the on-axis-video system in a protein well of plate B. (e) The image of crystal growth under different crystallization conditions with plate A.

(f) The image of crystal growth under different crystallization conditions with plate B.

\subsection{In Situ Crystal Location}

Once crystals were grown in microplate A or B, microplates were mounted on the goniometer head of the MD2 diffractometer for data collection, and it was important to quickly achieve precise alignment between the microcrystals and the incident X-rays. Unlike many other in situ data collection with traditional plates systems, our microplates can be moved into the beam position without adding any other motor systems. Moreover, crystals can be clearly focused to the beam position with the beamline centering system. Figure $3 \mathrm{a}, \mathrm{b}$ shows the typical working position of microplates A and B at the beamline. However, it is extremely difficult to align the crystal to beam position with normal 96-well crystal plates, because normal sized crystal plates are difficult to rotate $90^{\circ}$ to align the crystal to the beam position. 

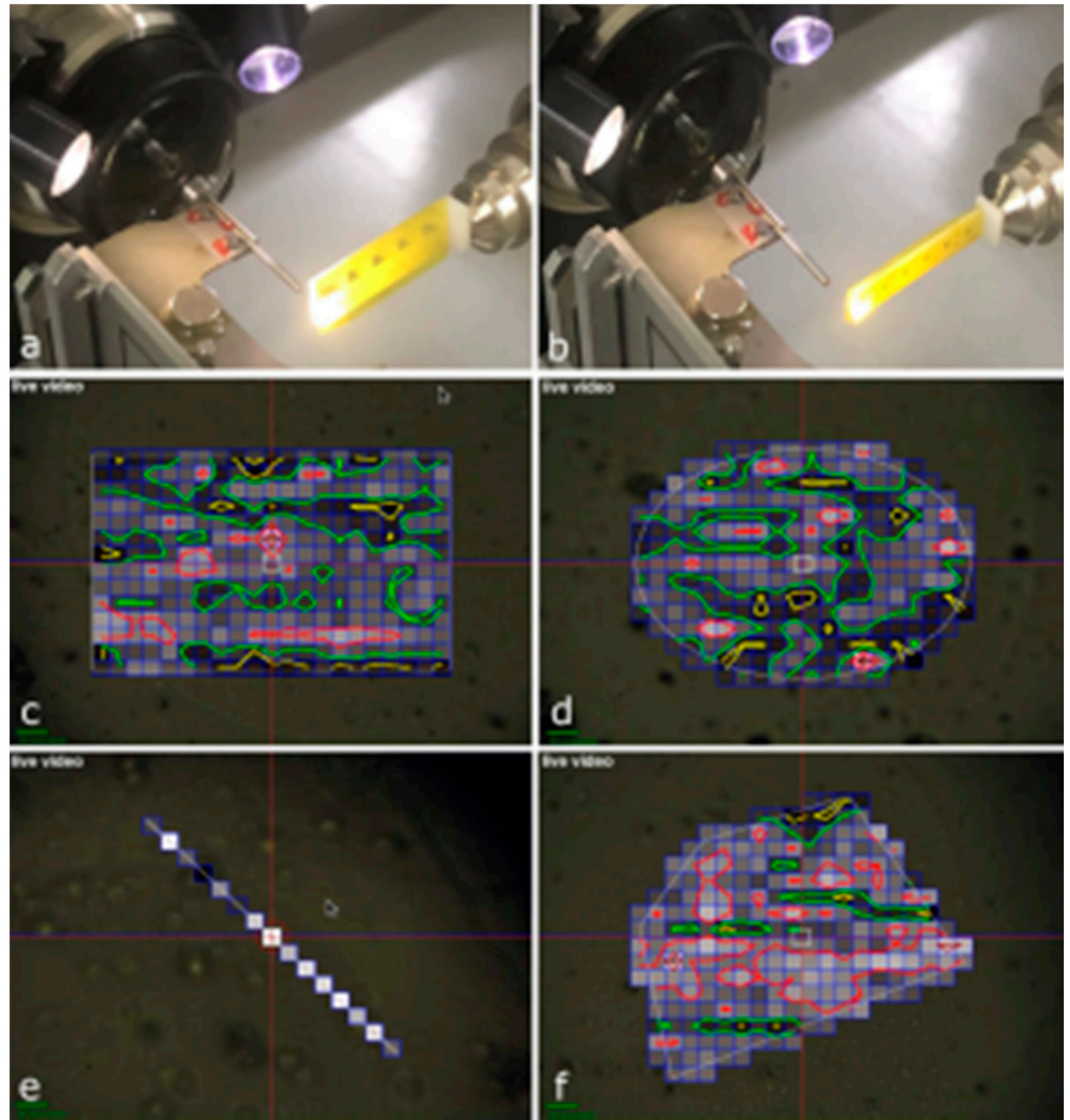

Figure 3. Microplates mounted on the goniometer head for data collection. (a) Plate A with 10 protein wells was installed on the goniometer head. (b) Plate B with 18 protein wells was installed on the goniometer head. Crystal location using different grid scanning types: (c) rectangle scanning area; (d) oval scanning area; (e) line scanning area; (f) polygon scanning area.

Crystal location results using grid scanning with plate B under cryogenic temperature are shown in Figure 3. Our results show that the crystal position, located on the Kapton membrane, can be easily identified by grid scanning. Four grid scanning types (rectangle, oval, line, polygon) can be used for plate B to promote the crystal location, the grid size, rotation wedge, and exposure time that can be defined by the user. The Kapton membrane does not affect the crystal location by grid scanning, moreover, to confirm that there is no effect of Kapton membrane on the crystal location, the effect of Kapton membrane is investigated in Section 3.3.

The combined use of the in situ diffraction plate and grid scan significantly improves the efficiency of diffraction data screening and collection. The common data collection of crystals requires each crystal to be separately installed on the goniometer, centered under the X-ray irradiation, and then removed and the sample changed. For this experiment, there are hundreds of lysozyme microcrystals in a protein well, and a set of plates can be loaded with thousands of crystals at the same time. Grid scanning can scan the entire protein well at once, and then it can quickly determine the position of all crystals in a protein well and the preliminary diffraction value of that position will be displayed. 
Click the grid point with high diffraction value, and the system will automatically align it with the optical path, which can greatly reduce the time of sample exchange and positioning.

\subsection{Background Scattering}

Resolution is used as an important parameter for evaluating the quality of diffraction data and analyzing the effect of background scattering. The highest-resolution shells are determined using the following criteria: signal-to-noise ratio $[\mathrm{I} / \sigma(\mathrm{I})]>2$, redundancy $>2$, completeness $>85 \%$, and Rmerge $<1$.

The background scattering of the crystal support material will affect the quality of the diffraction data of the protein crystal. When the background scattering of the support material is large, it will increase the noise points of the diffraction data, thereby masking the signal intensity of the diffraction points of the protein crystal. The selection of the material of the crystal support film requires special consideration: (1) High temperature resistance; high temperature will be generated after X-ray irradiation of the film material, which is likely to cause the film of some materials to be burnt and deformed. (2) High transparency; thin film materials with low light transparency are not conducive to the positioning of microcrystals. Thin films with high transparency can make the crystals visible on the microscope on the beamline station, which can efficiently locate the crystal position and facilitate data collection. (3) Chemical corrosion resistance; the crystal solution may contain corrosive components, or may easily chemically react with some thin film materials, resulting in the thin film materials being unusable. (4) Radiation resistance; synchrotron radiation $X$-ray has a high luminous flux, so the radiation dose is large, and the selected thin film material should be radiation resistant. At present, the commonly used films for serial crystallographic experiments based on fixed targets include Maylar film, polyimide film, synthetic cyclic olefin copolymer (COC), polycarbonate plastic, and other materials. Mylar film is a kind of polyester film with good light transmittance. The COC film is resistant to high temperature and chemical corrosion. Polyester film has a high melting point and good light transmission performance. The polyimide membrane, for example Kapton membrane, has many advantages and is best suited to all the requirements of our experiment, it is a kind of membrane with high temperature resistance, good light transmission performance, and chemical corrosion resistance. When the energy is $9-15 \mathrm{keV}$, the $\mathrm{X}$-ray transmittance of $12.5 \mu \mathrm{m}$ thick polyimide film can reach $99 \%$, and it produces low background scattering under X-ray irradiation. Polyimide film has very good light transmittance, and microcrystals of approximately $10 \mu \mathrm{m}$ are visible under the microscope. Therefore, it is very easy to locate the position of the crystal at the beamline station. Here, we only use Kapton membrane for our experiment.

In order to explore the influence of the background scattering of the in situ device including the crystallization drops and Kapton membranes on the quality of the protein crystal diffraction data, the air diffraction image (Figure 4a) and the in situ device diffraction image (Figure 4b) were collected respectively. Comparing the background scattering of air with the in situ device, it can be found that there is little difference between the two experiments (Figure 4a,b). In order to further prove the influence of the in situ device on the analysis of diffraction data, we collected the diffraction data of microcrystals by using the in situ device and a nylon loop. From the results of the resolution signal-to-noise ratio data (Figure 4c,d). It can be seen that there is little difference between the in situ device and the nylon loop. The in situ diffraction device has a major influence on the signal-to-noise ratio of the crystal diffraction data at approximately $4 \AA$, but the difference is basically negligible. The results show that the method of loading a microcrystal with the in situ device can also obtain high-quality diffraction data. The in situ device has lower background scattering and will not affect the quality of the crystal data. 

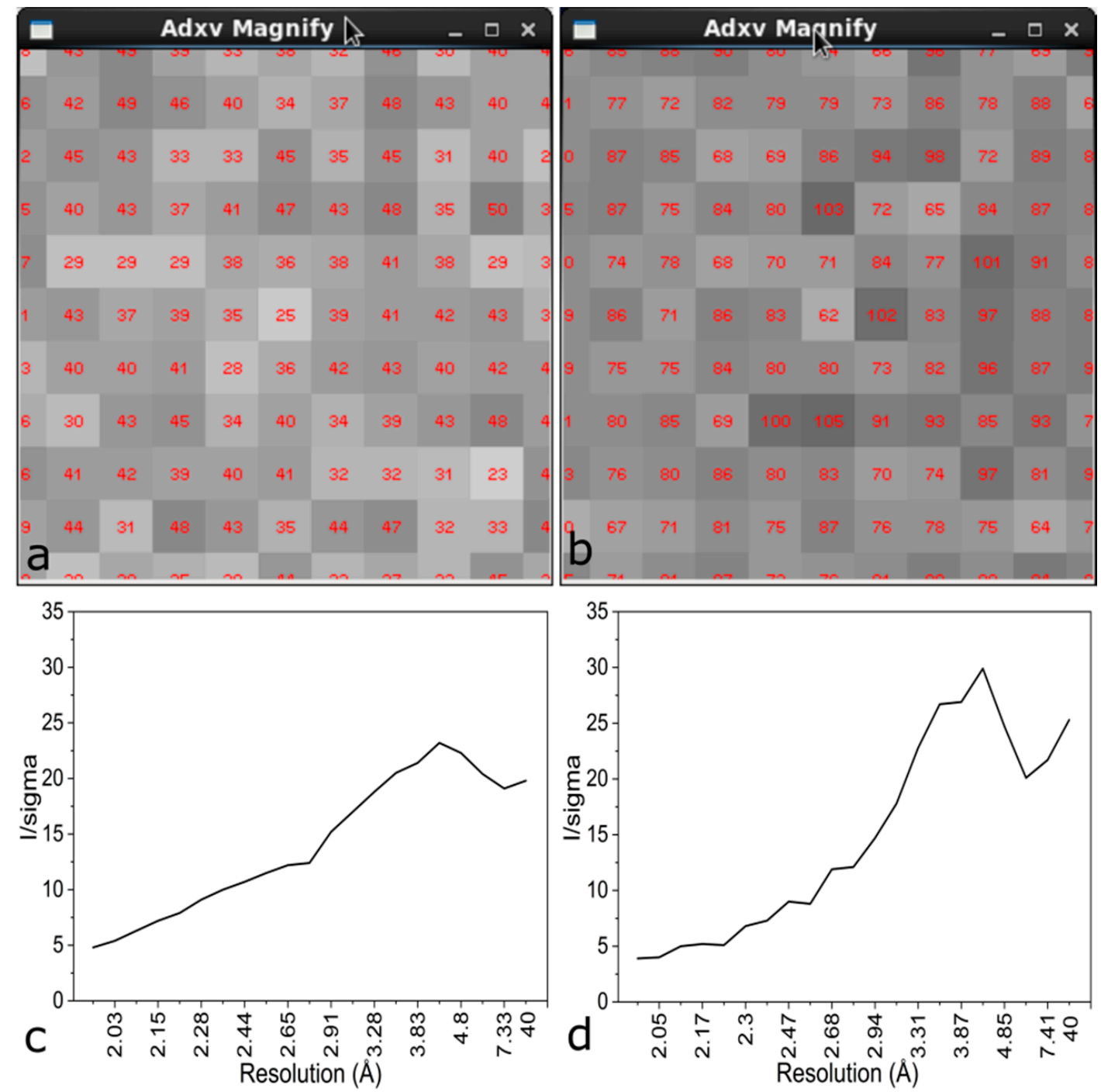

Figure 4. Background noise of air (a) and the in situ device (b) derived from the same coordinate point of the two diffraction patterns. (c) Signal-to-noise ratio in every resolution shell of the diffraction data obtained from a single lysozyme crystal in the nylon loop. (d) Signal-to-noise ratio in every resolution shell of the diffraction data obtained from multiple lysozyme crystals in the microplate.

\subsection{Structure Determination with Microcrystals}

We used lysozyme microcrystals (less than $20 \mu \mathrm{m}$ ) to verify the in situ microplates, and the data was collected at the BL18U beamline station of SSRF. When performing the experiments at room temperature, we used the visual method (Figure 2) to select 20 crystals from plate A for diffraction data collection. According to the radiation dose limit, five diffraction images were collected for each crystal (typical pattern in Figure 5b), and the relatively poor diffraction images were deleted, then each set of data was indexed with XDS. In the end, we selected five data sets with a total of 25 images, and these images were integrated through the blend program and then used for further structural analysis. According to the above-mentioned highest resolution criteria, we finally got the highest resolution of $2.15 \AA$. When performing the experiments at cryogenic temperatures, we used grid scanning (Figure 3) to select 10 crystals from plate B for diffraction data collection. A total of 40 diffraction images were collected from each crystal (typical pattern in Figure $5 \mathrm{c}$ ). The data processing is the same as above. Finally, four data sets with 40 images were integrated through the blend program and then we got the highest resolution of $1.98 \AA$. 


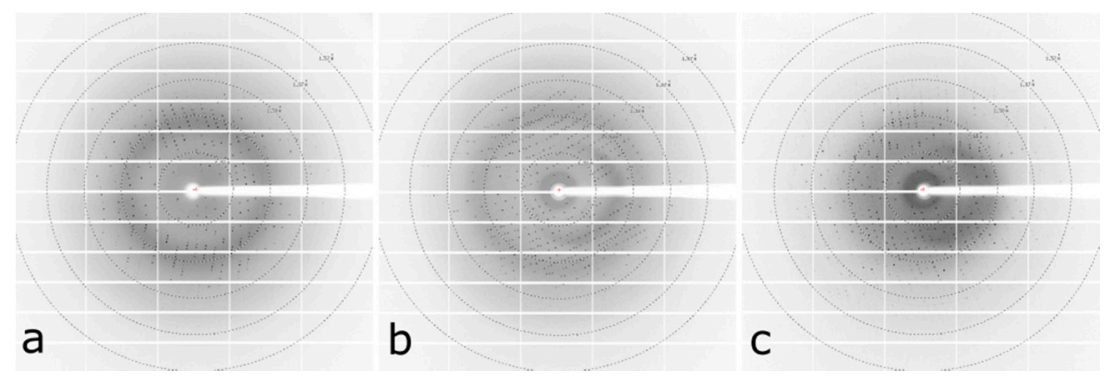

Figure 5. Typical diffraction pattern collected from crystal mounted by Nylon loop at $100 \mathrm{~K}$ (a) and the microplate A at room temperature (b) and the microplate B at $100 \mathrm{~K}$ (c).

In order to compare with the common method, the nylon loop was used for diffraction data collection. We used the same method to cultivate lysozyme microcrystals, and then the same data acquisition strategy was used to collect data from lysozyme at cryogenic temperature. According to the aforementioned data processing method, we finally integrated 60 diffraction images (typical pattern in Figure 5a) and obtained the highest resolution of $1.96 \AA$. The comparison of data collection through the in situ microplates and the data collection through the nylon loop is shown in Table 1.

Table 1. Statistical analysis of lysozyme using a nylon loop and the in situ plate for data collection. The data collection for multiple crystals was performed at a low temperature (100 K) and room temperature (RT), respectively.

\begin{tabular}{|c|c|c|c|}
\hline & Nylon Loop (100 K) & Microplates (RT) & Microplates (100 K) \\
\hline \multicolumn{4}{|c|}{ Data collection } \\
\hline Dominant size in sample $(\mu \mathrm{m})$ & $<20$ & $<20$ & $<20$ \\
\hline Number of data sets & 1 & 5 & 4 \\
\hline Number of images & 60 & 25 & 40 \\
\hline Space group & P 422 & P 422 & P 422 \\
\hline \multicolumn{4}{|c|}{ Unit cell } \\
\hline$a, b, c(\AA)$ & 79.3379 .3336 .94 & 79.2179 .2137 .83 & 79.8079 .8036 .96 \\
\hline$a, b, c\left(^{\circ}\right)$ & 90.0090 .0090 .00 & 90.0090 .0090 .00 & 90.0090 .0090 .00 \\
\hline Energy (keV) & 12 & 12 & 12 \\
\hline Resolution range $(\AA)$ & $39.66-1.96$ & $37.83-2.15$ & $39.90-1.98$ \\
\hline Number of unique reflections & 8169 & 6796 & 7439 \\
\hline Completeness (\%) & $94.9(86.9)$ & 97.5(95.9) & $87.7(84.6)$ \\
\hline Rmerge (\%) & $4.5(46.4)$ & $10.8(88.0)$ & $9.6(52.0)$ \\
\hline$<\mathrm{I} / \sigma(\mathrm{I})>$ & $19.8(4.8)$ & $8.8(2.0)$ & $25.3(3.9)$ \\
\hline Redundancy & $4.5(3.9)$ & $3.6(2.9)$ & $2.5(3.1)$ \\
\hline
\end{tabular}

In this experiment, we developed an efficient method for sample delivery and data collection for multicrystals. A Kapton membrane was utilized for crystal growth and sealing up the microplate. A complete dataset can be obtained after merging multiple datasets and the structure can be solved. Comparing the in situ microplates with the single nylon loop, the data collected using the in situ microplates at cryogenic temperature have the same good quality as the data collected using a single nylon loop, but the data collected using the in situ microplates at room temperature demonstrate worse resolution and signal-to-noise ratio, which is because the quality of crystals is affected at room temperature (Table 1). Moreover, electronic density comparation shows that there is no significant difference between results from nylon loop at $100 \mathrm{~K}$ and those from microplate $\mathrm{B}$ at $100 \mathrm{~K}$. However, we do observe that there is a slight disappearance of electronic density obtained from microplate A at room temperature (F38 blue), compared with those from the nylon loop at $100 \mathrm{~K}$ (F38 green) or microplate B at $100 \mathrm{~K}$ (F38 red) (Figure 6). Our signal-to-noise analysis and electronic density analysis show that the quality of crystals is affected by radiation damage at room temperature. However, the discrepancy does not significantly affect the results. 


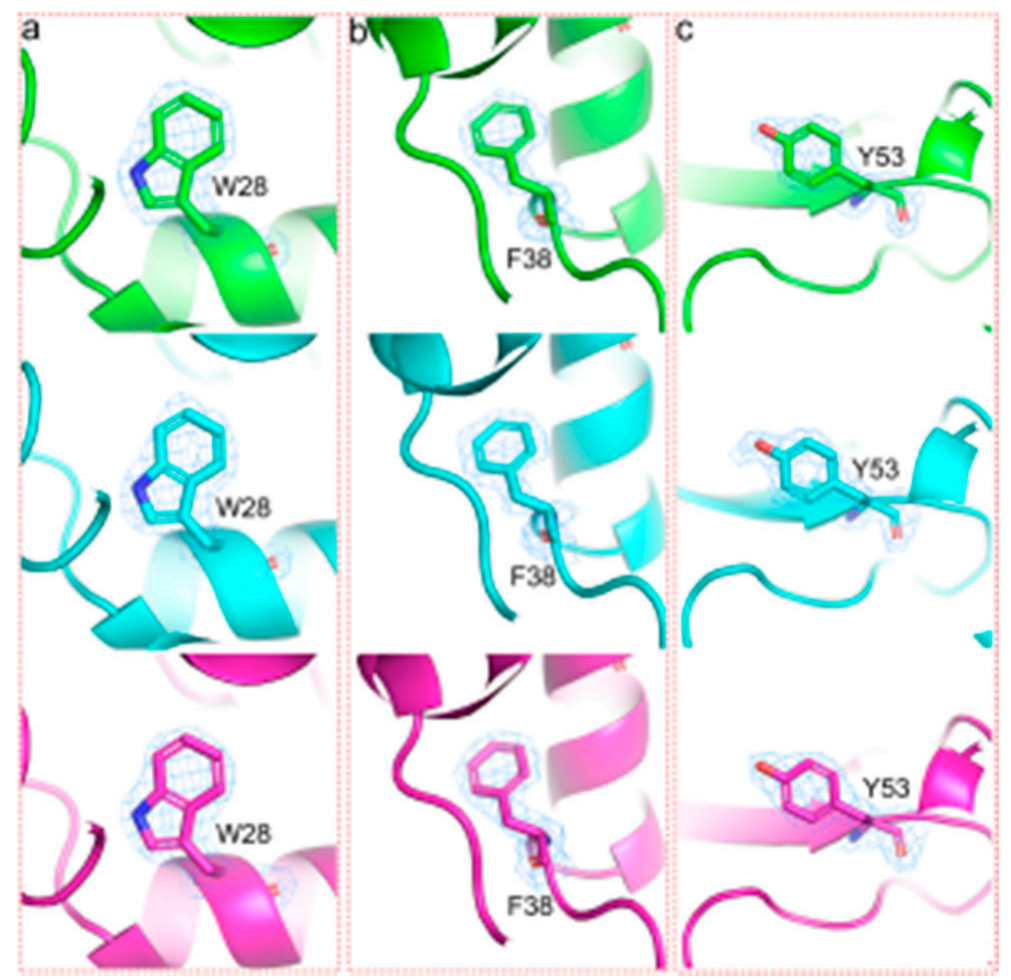

Figure 6. Enlargement of the three typical lysozyme residues and view of the extra electron density observed after partial refinement using the data set collected from crystal mounted by the Nylon loop at $100 \mathrm{~K}$ (green) and microplate A at room temperature (blue) and microplate B at $100 \mathrm{~K}$ (red). (a) View of Lysozyme W28 residue. (b) View of Lysozyme F38 residue. (c) View of Lysozyme Y57 residue.

In summary, this new method based on microplates realizes the in situ growth and simultaneous sample loading of multiple crystals, and it also realizes the rapid localization of crystals and the efficient data collection. On the premise that the in situ device has little effect on the data quality and low background scattering, we obtained comparable data quality to that of the traditional method with the nylon loop.

The in situ microplate including plate A and plate B has three advantages compared to the common commercially available in situ plate. First of all, except for the side parts of the microplate, the in situ microplate can almost perform a 360-degree data collection on the crystal without deviating from the optical path, and it can also be rotated at a full angle for rapid centering. The second is that the microplates are easy to manufacture and operate. The manual loading operation of the in situ microplate is the same as that of the ordinary nylon loop, which means that extra motors are not required, and it will not be limited by extra motors in most cases. The high-resolution structure of the protein can be obtained, and the manufacture of the in situ plate is fully customizable, which is suitable for most beamline stations. Third, the in situ microplates can support the cultivation of one protein under multiple crystallization conditions and the in situ cultivation of multiple proteins. This is not only suitable for the integration of multiple data sets of microcrystals, but also for multiple sets of data collection for multiple large crystals. Our microplate can save time for changing samples, and has the function of screening crystallization conditions.

However, the in situ microplates also have the following problems. When the in situ microplates are used to screen protein crystallization conditions, few crystallization conditions can be screened at a time, and it is only suitable for the fine screening of protein crystallization conditions. The size of these in situ microplates is affected by the limitation of the motor of the SSRF beamline BL18U1. In order to reduce costs, we use low-precision 3D printing technology and choose white resin materials, so far, we manually add protein samples to each well, therefore, the size of the protein wells are larger 
than chip device, the distance between the two protein wells is also longer than chip device. If the researcher needs to screen more protein crystallization conditions, the in situ microplates can also be designed to have more protein wells and crystallization chambers by adopting mechanical spotting or using higher-precision processing technology, but the design of the in situ microplates needs to meet requirements mentioned in the previous section.

Furthermore, the automation level of the fixed target serial crystallography method based on the thin film in this research is far from enough, and the automatic sample delivery and data collection of crystals is still not fully automatic. In the future, the automatic data collection of crystals should be further improved. At the same time, these methods have not yet resolved protein crystals of unknown structure. These methods should be used to further test protein crystals of unknown structure to improve these methods.

\section{Conclusions}

In recent years, there has been a growing interest in collecting X-ray diffraction data directly from the crystallization plates to eliminate the crystal harvesting step. The use of the microplates and the grid scanning at SSRF enables efficient data collection and crystallization condition screening. Hundreds of crystals can be mounted on the goniometer head simultaneously by microplates, which bypasses the tedious step of mounting single crystals by a user or robot, and microcrystals can be efficiently aligned to the beam position for data collection. Additionally, the experiments can be conducted at room temperature and cryogenic temperature. Furthermore, this method is particularly attractive for fragile or optically invisible microcrystals. Our microplates are suitable for data collection for solving structures of multiple crystals at room temperature or cryogenic temperature.

Author Contributions: Conceptualization, Z.W.; Data curation, M.L. and H.W.; Funding acquisition, J.H.; Methodology, M.L. and L.Y.; Project administration, Q.W. and J.H.; Resources, B.S., H.Z., F.Y., Q.W. and J.H.; Software, Z.W.; Supervision, L.Y., B.S., H.Z., F.Y. and Q.W.; Validation, M.L.; Visualization, H.W.; Writing-original draft, M.L.; Writing-review and editing, Z.W. All authors have read and agreed to the published version of the manuscript.

Funding: This research was funded by The National Key Research and Development Program of China (Grant No. 2017YFA0504901).

Acknowledgments: The authors thank the teams at the BL18U1 beamline of the Shanghai Synchrotron Radiation Facility for the beamtime allocated to this project and for their help in experiments.

Conflicts of Interest: The authors declare no conflict of interest.

\section{References}

1. Mcpherson, A. In situ X-ray crystallography. J. Appl. Crystallogr. 2010, 33, 397-400. [CrossRef]

2. Heymann, M.; Opthalage, A.; Wierman, J.L.; Akella, S.; Szebenyi, D.M.E.; Gruner, S.M.; Fraden, S. Room-temperature serial crystallography using a kinetically optimized microfluidic device for protein crystallization and on-chip X-ray diffraction. IUCrJ 2014, 1, 349-360. [CrossRef] [PubMed]

3. Li, L.; Mustafi, D.; Fu, Q.; Tereshko, V.; Chen, D.L.L.; Tice, J.D.; Ismagilov, R.F. Nanoliter microfluidic hybrid method for simultaneous screening and optimization validated with crystallization of membrane proteins. Proc. Nat. Acad. Sci. USA 2006, 103, 19243-19248. [CrossRef] [PubMed]

4. Perry, S.L.; Guha, S.; Pawate, A.S.; Henning, R.; Kosheleva, I.; Šrajer, V.; Kenis, P.J.A.; Ren, Z. In situserial Laue diffraction on a microfluidic crystallization device. J. Appl. Crystallogr. 2014, 47, 1975-1982. [CrossRef]

5. Kisselman, G.; Qiu, W.; Romanov, V.; Thompson, C.M.; Lam, R.; Battaile, K.P.; Pai, E.F.; Chirgadze, N.Y. X-CHIP: An integrated platform for high-throughput protein crystallization and on-the-chip X-ray diffraction data collection. Acta Crystallogr. Sect. D Boil. Crystallogr. 2011, 67, 533-539. [CrossRef]

6. Bingel-Erlenmeyer, R.; Olieric, V.; Grimshaw, J.P.A.; Gabadinho, J.; Wang, X.; Ebner, S.G.; Isenegger, A.; Schneider, R.; Schneider, J.; Glettig, W.; et al. SLS Crystallization Platform at Beamline X06DA-A Fully Automated Pipeline Enablingin SituX-ray Diffraction Screening. Cryst. Growth Des. 2011, 11, 916-923. [CrossRef] 
7. Pinker, F.; Brun, M.; Morin, P.; Deman, A.-L.; Chateaux, J.-F.; Oliéric, V.; Stirnimann, C.; Lorber, B.; Terrier, N.; Ferrigno, R.; et al. ChipX: A Novel Microfluidic Chip for Counter-Diffusion Crystallization of Biomolecules and in Situ Crystal Analysis at Room Temperature. Cryst. Growth Des. 2013, 13, 3333-3340. [CrossRef]

8. Pineda-Molina, E.; Daddaoua, A.; Krell, T.; Ramos, J.L.; Delgado-López, J.M.; Gavira, J.A. In situ X-ray data collection from highly sensitive crystals of Pseudomonas putida PtxS in complex with DNA. Acta Crystallogr. Sect. F Struct. Boil. Cryst. Commun. 2012, 68, 1307-1310. [CrossRef]

9. Yadav, M.K.; Gerdts, C.J.; Sanishvili, R.; Smith, W.W.; Roach, L.S.; Ismagilov, R.F.; Kuhn, P.; Stevens, R.C. In situdata collection and structure refinement from microcapillary protein crystallization. J. Appl. Crystallogr. 2005, 38, 900-905. [CrossRef]

10. Solvas, X.C.I.; Demello, A. Droplet microfluidics: Recent developments and future applications. Chem. Commun. 2011, 47, 1936-1942. [CrossRef]

11. Maeki, M.; Yoshizuka, S.; Yamaguchi, H.; Kawamoto, M.; Yamashita, K.; Nakamura, H.; Miyazaki, M.; Maeda, H. X-ray diffraction of protein crystal grown in a nano-liter scale droplet in a microchannel and evaluation of its applicability. Anal. Sci. 2012, 28, 65. [CrossRef] [PubMed]

12. Roedig, P.; Duman, R.; Sanchez-Weatherby, J.; Vartiainen, I.; Burkhardt, A.; Warmer, M.; David, C.; Wagner, A.; Meents, A. Room-temperature macromolecular crystallography using a micro-patterned silicon chip with minimal background scattering. J. Appl. Crystallogr. 2016, 49, 968-975. [CrossRef] [PubMed]

13. Mueller, C.; Marx, A.; Epp, S.W.; Zhong, Y.; Kuo, A.; Balo, A.R.; Soman, J.; Schotte, F.; Lemke, H.T.; Owen, R.L.; et al. Fixed target matrix for femtosecond time-resolved and in situ serial micro-crystallography. Struct. Dyn. 2015, 2, 054302. [CrossRef]

14. Murray, T.D.; Lyubimov, A.Y.; Ogata, C.M.; Vo, H.; Uervirojnangkoorn, M.; Brünger, A.T.; Berger, J.M. A high-transparency, micro-patternable chip for X-ray diffraction analysis of microcrystals under native growth conditions. Acta Crystallogr. Sect. D Boil. Crystallogr. 2015, 71, 1987-1997. [CrossRef] [PubMed]

15. Huang, C.Y.; Olieric, V.; Ma, P.K.; Panepucci, E.; Diederichs, K.; Wang, M.; Caffrey, M. In meso in situserial X-ray crystallography of soluble and membrane proteins. Acta Crystallogr. Sect. D Boil. Crystallogr. 2015, 71, 1238-1256. [CrossRef]

16. Roedig, P.; Vartiainen, I.; Duman, R.; Panneerselvam, S.; Stübe, N.; Lorbeer, O.; Warmer, M.; Sutton, G.; Stuart, D.I.; Weckert, E.; et al. A micro-patterned silicon chip as sample holder for macromolecular crystallography experiments with minimal background scattering. Sci. Rep. 2015, 5, 10451. [CrossRef]

17. Zarrine-Afsar, A.; Barends, T.R.M.; Müller, C.; Fuchs, M.R.; Lomb, L.; Schlichting, I.; Miller, R.J.D. Crystallography on a chip. Acta Crystallogr. Sect. D Boil. Crystallogr. 2012, 68, 321-323. [CrossRef]

18. Li, B.; Huang, S.; Pan, Q.Y.; Li, M.-J.; Zhou, H.; Wang, Q.-S.; Yu, F.; Sun, B.; Chen, J.-Q.; He, J.-H. New design for multi-crystal data collection at SSRF. Nucl. Sci. Tech. 2018, 29, 21. [CrossRef]

19. Baxter, E.L.; Aguila, L.; Alonso-Mori, R.; Barnes, C.O.; Bonagura, C.A.; Brehmer, W.; Brünger, A.T.; Calero, G.; Caradoc-Davies, T.T.; Chatterjee, R.; et al. High-density grids for efficient data collection from multiple crystals. Acta Crystallogr. Sect. D Struct. Boil. 2016, 72, 2-11. [CrossRef]

20. Michalska, K.; Tan, K.; Chang, C.; Li, H.; Hatzos-Skintges, C.; Molitsky, M.; Alkire, R.; Joachimiak, A. In situ X-ray data collection and structure phasing of protein crystals at Structural Biology Center 19-ID. J. Synchrotron Radiat. 2015, 22, 1386-1395. [CrossRef]

21. McPhillips, T.M.; McPhillips, S.E.; Chiu, H.J.; Cohen, A.E.; Deacon, A.M.; Ellis, P.J.; Garman, E.; Gonzalez, A.; Sauter, N.K.; Phizackerley, R.P.; et al. Blu-Ice and the Distributed Control System: Software for data acquisition and instrument control at macromolecular crystallography beamlines. J. Synchrotron Radiat. 2002, 9, 401-406. [CrossRef] [PubMed]

22. Owen, R.L.; Rudino-Pinera, E.; Garman, E.F. Experimental determination of the radiation dose limit for cryocooled protein crystals. Proc. Natl. Acad. Sci. USA 2006, 103, 4912-4917. [CrossRef]

23. Krug, M.; Weiss, M.S.; Heinemann, U.; Mueller, U. XDSAPP: A graphical user interface for the convenient processing of diffraction data using XDS. J. Appl. Crystallogr. 2012, 45, 568-572. [CrossRef]

24. Foadi, J.; Aller, P.; Alguel, Y.; Cameron, A.D.; Axford, D.; Owen, R.L.; Armour, W.; Waterman, D.G.; Iwata, S.; Evans, G. Clustering procedures for the optimal selection of data sets from multiple crystals in macromolecular crystallography. Acta Crystallogr. Sect. D Boil. Crystallogr. 2013, 69, 1617-1632. [CrossRef]

25. Collaborative, C.P. The CCP4 suite: Programs for protein crystallography. Acta Crystallogr. Sect. D Biol. Crystallogr. 1994, 50, 760. [CrossRef] 
26. Adams, P.D.; Grosse-Kunstleve, R.W.; Hung, L.W.; Ioerger, T.R.; McCoy, A.J.; Moriarty, N.W.; Read, R.J.; Sacchettini, J.C.; Sauter, N.K.; Terwilliger, T.C. PHENIX: Building new software for automated crystallographic structure determination. Acta Crystallogr. Sect. D Boil. Crystallogr. 2002, 58, 1948-1954. [CrossRef] [PubMed]

27. Emsley, P.; Cowtan, K. Coot: Model-building tools for molecular graphics. Acta Crystallogr. Sect. D Biol. Crystallogr. 2004, 60, 2126-2132. [CrossRef] [PubMed]

(C) 2020 by the authors. Licensee MDPI, Basel, Switzerland. This article is an open access article distributed under the terms and conditions of the Creative Commons Attribution (CC BY) license (http://creativecommons.org/licenses/by/4.0/). 